\title{
PU.1 Is Identified as a Novel Metastasis Suppressor in Hepatocellular Carcinoma Regulating the miR-615-5p/IGF2 Axis
}

\author{
Li-Jie Song ${ }^{\circledR}$, Wei-Jie Zhang ${ }^{\star}$, Zhi-Wei Chang, Yan-Feng Pan, Hong Zong, Qing- \\ Xia Fan, Liu-Xing Wang*
}

\begin{abstract}
Invasion and metastasis is the major cause of tumor recurrence, difficulty for cure and low survival rate. Excavating key transcription factors, which can regulate tumor invasion and metastasis, are crucial to the development of therapeutic strategies for cancers. PU.1 is a master hematopoietic transcription factor and a vital regulator in life. Here, we report that, compared to adjacent non-cancerous tissues, expression of PU.1 mRNA in metastatic hepatocellular carcinoma (HCC), but not primary HCC, was significantly down-regulated. In addition, levels of PU.1 mRNA in metastatic hepatoma cell lines MHCC97L and MHCC97H were much lower than in non-metastatic Hep3B cells. Transwell invasion assays after PU.1 siRNA transfection showed that the invasion of hepatoma cell lines was increased markedly by PU.1 knockdown. Oppositely, overexpression of PU.1 suppressed the invasion of these cells. However, knockdown and overexpression of PU.1 did not influence proliferation. Finally, we tried to explore the potential mechanism of PU.1 suppressing hepatoma cell invasion. ChIP-qPCR analysis showed that PU.1 exhibited a high binding capacity with miR-615-5p promoter sequence. Overexpression of PU.1 caused a dramatic increase of pri-, pre- and mature miR-615-5p, as well as a marked decrease of miR-615-5p target gene IGF2. These data indicate that PU.1 inhibits invasion of human HCC through promoting miR-615-5p and suppressing IGF2. These findings improve our understanding of PU.1 regulatory roles and provided a potential target for metastatic HCC diagnosis and therapy.
\end{abstract}

Keywords: PU.1 - miR-615-5p - hepatocellular carcinoma - invasion and metastasis

Asian Pac J Cancer Prev, 16 (9), 3667-3671

\section{Introduction}

Hepatocellular carcinoma (HCC) is one of the most leading causes of cancer-related deaths globally (Kudo et al., 2014). As a typical malignant tumor, HCC caused high mortality rate owing to frequent tumor invasion and metastasis, and tumor recurrence after surgical resection (Bruix and Sherman, 2011; Llovet and Hernandez-Gea, 2014). Invasion and metastasis is the major cause of tumor recurrence, difficulty for cure and low survival rate (Toiyama et al., 2014).

In the last two decades, a large number of evidence indicated that microRNAs (miRNAs) played an important role in multiple biological processes, including development of normal and cancerous tissues (Drusco et al., 2014). In mammals, miRNAs form imperfect matches with the 3' untranslated regions (UTRs) of their target mRNAs and cause mRNA translational repression (Olena and Patton, 2014). Down-regulating their target gene expression, miRNAs cause aberrant gene expression in cancer initiation and progression. Multiple miRNAs have been implicated in the development of HCC. Let-7, one of the primitively identified miRNAs, was reported to suppress expression of anti-apoptotic protein Bcl-xL and potentiated sorafenib-induced apoptosis in human HCC (Shimizu et al., 2010; Guan, 2015). Liver-specific miR-122 acts as a tumor-suppressor by down-regulating cyclin G1 and subsequently enhancing p53 transcriptional activity (Kutay et al., 2006; Fornari et al., 2009; Liu et al., 2014). Most recently, miR-615-5p, a miRNA which has been poorly characterized since it was found, was identified to be restrictedly expressed in cirrhotic and cancerous liver tissues and alleviated the tumorigenic effects in HCC through targeting insulin-like growth factor 2 (IGF2) (Tayebi et al., 2012). Excavating key transcription factors, which contribute to regulating expression of these miRNAs, is crucial to the development of therapeutic strategies for metastatic HCC. However, with respect to mature miRNAs and their target genes, little is known to their upstream expression-regulating genes.

The transcription factor PU.1 (PU box binding protein, 


\section{Li-Jie Song et al}

also spleen focus forming virus proviral integration oncogene, spi1) is a hematopoietic ETS family member that plays a key role in the development of congenital and acquired immune system (Wei et al., 2014; Christie et al., 2015). As a vital transcription factor, PU.1 whole body deficient mice exhibited early fetal death (Metcalf et al., 2006). Numerous downstream genes were regulated by PU.1 in a cell-intrinsic manner and even modest variations in PU.1 expression may lead to robust up- or down-regulation of gene expression (Wei et al., 2014). PU.1 function has been restricted in hematopoietic system, until recently, several studies indicated that PU.1 was expressed in mouse and human adipose tissues and played an negative role in adipogenesis through suppressing the adipogenic master gene peroxisome proliferator-activated receptor $\gamma$ (PPAR $\gamma$ ) (Wang and Tong, 2008; Pang et al., 2013; Wei et al., 2015). These studies suggested that regulatory role of PU.1 would not be confined in the hematopoietic system.

In this study, PU.1 was identified as an invasion suppressor in human HCC. Further ChIP-qPCR analysis and overexpression experiment showed that PU.1 was a transcription factor of miR-615-5p which was a newly characterized liver-specific tumor suppressor.

\section{Materials and Methods}

\section{Patients and sampling}

This study included 30 patients with primary HCC and 24 patients with metastatic HCC treated in our hospital. Each tumor tissue and its adjacent non-cancerous tissue were taken from the HCC patients. The study was approved by the Institutional Review Board of China. All participants gave their written informed consent.

\section{Cell culture}

Non-metastatic, low metastatic and high metastatic hepatoma cell lines Hep3B, MHCC97L and MHCC97H were purchased from Jingrong Biological Technology Co. Ltd. (Shanghai, China). The cells were taken from liquid nitrogen and then thawed in $37^{\circ} \mathrm{C}$ water bath. After centrifugated at $1000 \mathrm{~g}$ for $5 \mathrm{~min}$, the cells were suspended by dulbecco's modified eagle's medium (DMEM, containing $4.5 \mathrm{~g} / \mathrm{L}$ glucose, $4 \mathrm{mmol} / \mathrm{L}$ L-glutamine, Invitrogen, Carlsbad, CA) supplemented with $10 \%$ FBS (Invitrogen, Carlsbad, CA) and seeded into 12 well plates at the density of $10^{5} / \mathrm{cm}^{2}$. The cells were incubated in a humidified incubator with an atmosphere of $95 \%$ air-5\% $\mathrm{CO}_{2}$ at $37^{\circ} \mathrm{C}$.

\section{Transfection}

On reaching $80 \%$ of confluence, $1 \mu \mathrm{g}$ of pcDNA-PU.1, $1 \mu \mathrm{g}$ of pcDNA3.1 (vector), or 6 pM PU.1 siRNA were transfected alone or co- transfected into the $\mathrm{MHCC} 97 \mathrm{H}$ cell lines with Lipofectamine 3000 (Invitrogen) according to the manufacturer's instruction. The medium was changed every 3 days.

The pcDNA-PU.1 overexpression vector was kindly presented by Professor David Lohnes who are from Department of Cellular and Molecular Medicine, University of Ottawa. The 2'-OMe modified PU.1 siRNA were synthesized by Invitrogen company.

\section{Cell proliferation and invasion analysis}

Cell proliferation was evaluated using the MTT (Sigma, St Louis, MO) assay. Hep3B, MHCC97L and MHCC97H cells were seeded in 12-well culture plates at $0.5 \times 10^{5} /$ well, transfected with the indicated constructs or siRNA. The cells were incubated for $0,24,48,72$ and $96 \mathrm{~h}$ before adding the MTT reagent to each well at a final concentration of $0.5 \mathrm{mg} / \mathrm{ml}$ and incubated at $37^{\circ} \mathrm{C}$ for 4 h. After medium removal, $500 \mu \mathrm{l}$ of dimethyl sulfoxide was added to each well. Viable cells were measured by absorbance at a $550 \mathrm{~nm}$ wavelength using a microplate reader (BioRad, Hercules, CA).

Invasion were detected with a transwell migration and invasion assays. Cell invasion assay was performed with Matrigel (BD Biosciences, Sparks, MD) coated on the upper surface of the Transwell chamber (Corning, Lowell, MA). The cells that had migrated or invaded through the membrane were fixed with methanol and stained with crystal violet. Photographs of 3 randomly selected fields of the fixed cells were taken and cells were were measured by absorbance at a $550 \mathrm{~nm}$ wavelength using a microplate reader (BioRad, Hercules, CA).

\section{Real-time quantitative PCR}

Total RNA was isolated using TRIzol reagent (Invitrogen) following the manufacturer's instructions. The RNA concentration was quantified using a spectrophotometer measuring OD260/280 ratio (1.80$1.95)$. The integrity of RNA was checked by electrophoresis on $1.0 \%$ agarose gel with ethidium bromide staining. Realtime qPCR reactions were carried out in a final volume of $25 \mu$, using SYBR Premix Ex Taq (TaKaRa), 0.4 mM of each primer, and $200 \mathrm{ng}$ of cDNA template. Primers applied in the reactions are as follows: pri-miR-615-5p (5'- GCC AGC CAC CAA GAA GC-3', 5'-GCA GGG ATG CAG CGA TC-3'); pre-miR-615-5p (5'-GCT CCC GCT GTT TAC TCT G-3', 5'-AGG CTC TGG TGC TCC TGT C-3'); PU.1 (5' -TG GCT ATC ATT AAC CCT TAT-3', R: 5'-TTC TCC TGC CTC TTC CT-3'); IGF2 (5'-CCT CCT TTG GTC TTA CTG GG-3', R: 5'-CTT GGA CTT TGA GTC AAA TTG G-3'); $\beta$-actin (5'-ACG GGA CCT AAT GAA ACT C-3', R: 5'-CAA GAA GAT GCG GCT GT-3'); mature miR-615-5p stem-loop primer and quantitative primers were designed and produced by Ribobio company (Guangzhou, China). Each individual sample was run in triplicate wells. PCR amplification cycles were performed using iQTM5 Multicolor RealTime PCR Detection System (Bio-Rad) and SYBR Premix Ex Taq II kit (Invitrogen). The reactions were initially denatured at $95^{\circ} \mathrm{C}$ for 3 min followed by 35 cycles of $95^{\circ} \mathrm{C}$ for $15 \mathrm{~s}, 60^{\circ} \mathrm{C}$ for $1 \mathrm{~min}$. The change of transcript abundance of all tested genes was calculated using $2^{-\Delta \Delta \mathrm{Ct}}$ method. All mRNA amounts were normalized to $\beta$-actin.

\section{Western blotting}

Cells were lysed in lysis buffer (Beyotime) supplemented with $1 \mathrm{mM}$ PMSF. Protein concentration was determined with BCA protein assay kit (Tiangen). Twenty micrograms of protein of each sample were 
PU.1 Identified as a Novel Metastasis Suppressor in Hepatocellular Carcinoma Regulating miR-615-5p/IGF2 Axis

separated by $12 \%$ SDS-PAGE and electro-transferred to PVDF membrane (Millipore) for immunoblotting analysis. The following primary antibodies were used: Anti-PU.1 (1: 200, Abcam), Anti-IGF2 (1:300, Abcam), Anti- $\beta$-actin (1:200, Abcam) which was used as the internal reference. After incubation with the appropriate HRP-conjugate secondary antibody, proteins were detected using a ChemiDoc XRS imaging system and analysis software Quantity One (Bio-Rad).

\section{Website references}

The regulatory relationship between PU.1 and miR615-5p was calculated by the online platform ChIPBase (http://deepbase.sysu.edu.cn/chipbase/), which is an integrated resource for decoding transcription factor binding maps, expression profiles and transcriptional regulation of non-coding RNAs and protein-coding genes from ChIP-Seq data.

\section{ChIP-qPCR}

The cells were treated with $10 \%$ formaldehyde for 10 $\min$ at $37^{\circ} \mathrm{C}$. Then the cells were washed twice with icecold PBS and suspended in the lysis buffer $(0.5 \%$ NP-40, $150 \mathrm{mM} \mathrm{NaCl}, 50 \mathrm{mM}$ Tris, $\mathrm{pH} 7.5$, protease inhibitors). After 10 min incubation on ice, cells were centrifuged to pellet the nuclei. Nuclei were then suspended in the nuclei lysis buffer (1\% SDS, $10 \mathrm{mM}$ EDTA, $50 \mathrm{mM}$ Tris, $\mathrm{pH}$ $8.1, \mathrm{w} /$ protease inhibitors), incubated on ice for $10 \mathrm{~min}$ and sonicated for $1.5 \mathrm{~min}$ ( $30 \mathrm{~s}$ each time, then cooled on ice and repeated twice). The sheared chromatin was then immunoprecipitated by using anti-PU.1 antibody or IgG control. After extensive washing and then elution, the crosslink was reverted by heat treatment $\left(65^{\circ} \mathrm{C}\right.$ overnight and protease $\mathrm{K}$ digestion). The captured genomic DNA fragments were then purified by using Qiagen PCR purification kit. Identification of the captured Hox promoter fragments was performed by PCR analysis by using the promoter primers. The sequences of primers are 5'-CAAATA GCA GGC AAC AGT-3' (forward), 5'-ATA GAA GCAACACCT CCAC-3' (reverse). Thirty cycles of real-time PCR were performed using iQTM5 Multicolor Real-Time PCR Detection System (Bio-Rad) and SYBR Premix Ex Taq II kit (Invitrogen).

\section{Statistical analysis}

All data were obtained from at least three independent experiments. Values were expressed as means \pm SEM. Statistics were calculated with SPSS 18.0. Multiple comparisons were assessed by one-way ANOVA followed by Dunnett's tests. Difference between groups was considered statistically significant if $\mathrm{P}<0.05$.

\section{Results}

PU.1 mRNA was downregulated in human metastatic HCC tissues and metastatic HCC cell lines

Expression of PU.1 mRNA in cancerous tissues and their adjacent non-cancerous tissues from 30 patients with primary hepatoma were detected with real-time qPCR. The results exhibited that PU.1 mRNA levels showed no significance between the cancerous tissues and their adjacent ones (Figure 1A). Then, the same analysis was done in 24 patients with metastatic hepatoma. Interestingly, we found that PU.1 mRNA was downregulated in metastatic hepatomas compared to their adjacent non-cancerous tissues (Figure 1B). Subsequently, in Hep3B, MHCC97L and MHCC97H, which are repectively non-metastatic, low metastatic and high metastatic hepatoma cell lines, PU.1 mRNA levels were examined. The results showed that the mRNA level in metastatic cell lines MHCC97L and MHCC97H were much lower than that of non-metastatic Hep3B (Figure 1C). In addition, the mRNA level in high-metastatic cell line MHCC97H was much lower than that of lowmetastatic MHCC97L (Figure 1C). These data suggested that PU.1 might be associated with hepatoma invasion and metastasis.

Invasion of hepatoma cell lines was increased by transfection of PU.1 siRNA

To clarify the effect of PU.1 on hepatoma development in vitro, PU.1 siRNA was transfected into hepatoma cell lines Hep3B, MHCC97L and MHCC97H. After transfection for $48 \mathrm{~h}$, cell proliferation was detected with MTT assay and invasion was examined with a transwell invasion assay. The results showed that there was no significance in the proliferation of Hep3B, MHCC97L and $\mathrm{MHCC} 97 \mathrm{H}$ when PU.1 was knockdown compare to the control (Figure 2A-C), suggesting that silencing PU.1 did not influnce the proliferation of hepatoma cells. While in the absence of PU.1, the non-metastatic cell line Hep3B got some capacity of invasion (Figure 2D). Moreover, invasion of MHCC97L and MHCC97H was also significantly increased (Figure 2D).

Overexpression of PU.1 suppressed the invasion of hepatoma cells

Next, pcDNA-PU.1 expression vector was transfected into Hep3B, MHCC97L and MHCC97H cell lines. After

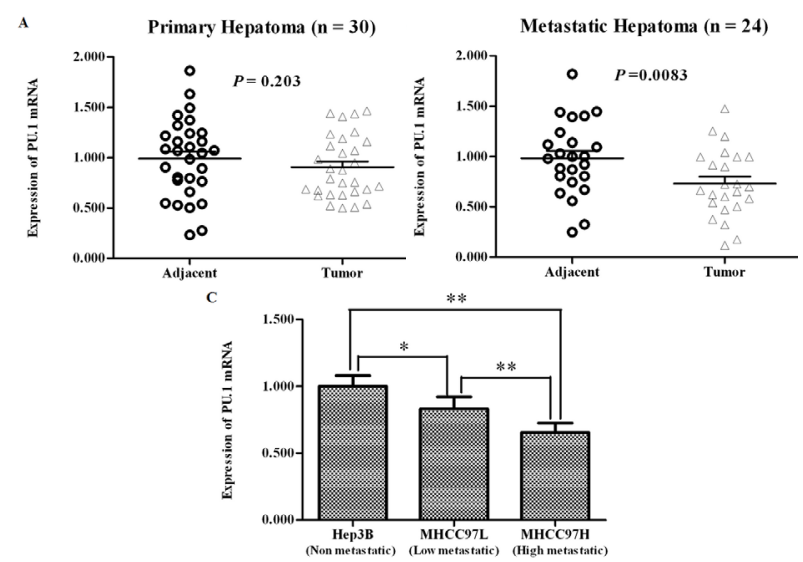

Figure 1. PU.1 was Lower Expressed in Metastatic HCC Tissues and Cell Lines. A) Comparison of PU.1 mRNA levels between primary $\mathrm{HCC}$ and adjacent non-cancerous tissues from 30 patients. B) Comparison of PU.1 mRNA levels between metastatic HCC and adjacent non-cancerous tissues from 24 patients. C) Comparison of PU.1 mRNA levels among Non-metastatic, low metastatic and high metas tatichepatoma cell lines Hep3B, MHCC97L and MHCC97H.* indicates significantly different $(P<0.05)$, ** indicates $P<0.01$ 
Li-Jie Song et al
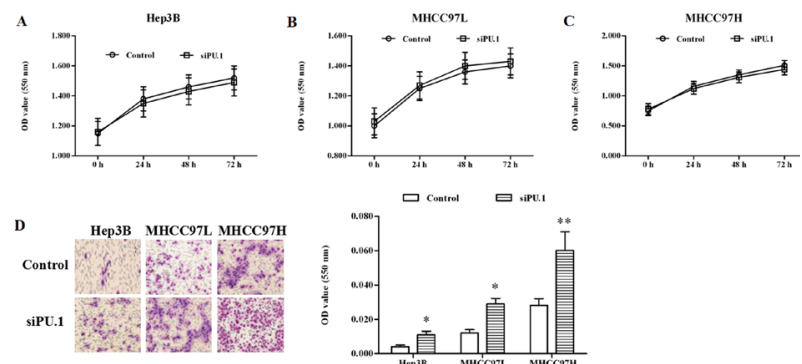

Figure 2. PU.1 knockdown promoted invasion of Hep3B, MHCC97L and MHCC97H cell lines. Proliferation of Hep3B A), MHCC97L B) and MHCC97H C) cell lines were detected with MTT approach at 0, 24, 4872 and $96 \mathrm{~h}$. D) Invasion were detected with a transwell migration and invasion assays after PU.1 siRNA transfection for $72 \mathrm{~h}$. The cells were were measured by absorbance at a $550 \mathrm{~nm}$ wavelength using a microplate reader. * indicates significantly different $(P<0.05)$, ** indicates $P<0.01$
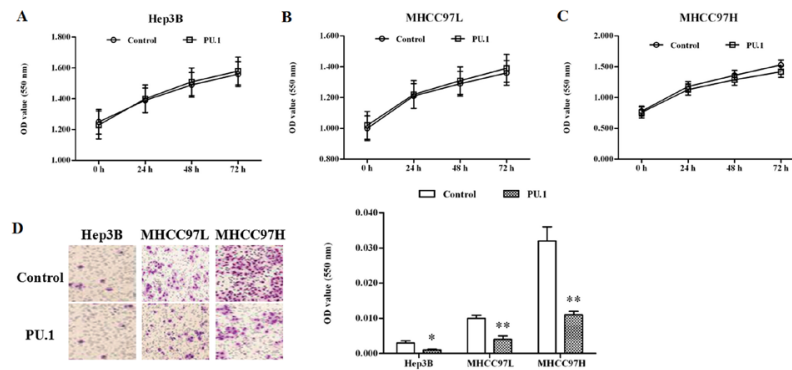

Figure 3. PU.1 Overexpression Promoted Invasion of Hep3B, MHCC97L and MHCC97H Cell Lines. Proliferation of Hep3B A), MHCC97L B) and MHCC97H C) cell lines were detected with MTT approach at 0, 24, 4872 and $96 \mathrm{~h}$. D) The cell invasion was detected with a transwell invasion assay after pcDNA3.1 and pcDNA-PU.1 transfection for 72 $\mathrm{h}$. The cells were were measured by absorbance at a $550 \mathrm{~nm}$ wavelength using a microplate reader. * indicates significantly different $(P<0.05)$, ** indicates $P<0.01$
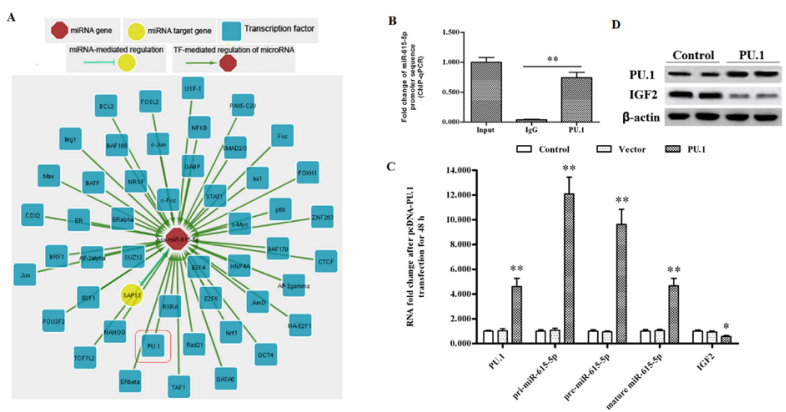

Figure 4. PU.1 was a Promoter of miR-615-5p in MHCC97H Cell Lines. A) The TF-miRNAs network integrated by online platform ChIPBase. B) Binding ability of PU.1 with promoter of miR-615-5p host gene Hox was checked by ChIP-qPCR analysis. C) RNA levels of pri-, pre-, mature miR-615-5p and its target gene IGF2 were increased by PU.1 overexpression. C. Protein level of miR-615-5p target gene IGF2 were increased by PU.1 overexpression. On reaching $80 \%$ of confluence, $1 \mu \mathrm{g}$ of pcDNA-PU.1 was transfected into the MHCC97H cell lines with Lipofectamine 3000. After transfection for $48 \mathrm{~h}$, expression of miR-615-5p and its target IGF were detected with real-time qPCR and Western blotting. $*$ indicates significantly different $(P<0.05), * *$ indicates $P<0.01$ transfection for $48 \mathrm{~h}$, cell proliferation and invasion were examined. The results showed that PU.1 overexpression did not influnce the proliferation of hepatoma cells (Figure 3A-C). Opposite to PU.1 siRNA transfection, invasion of metastatic hepatoma cell lines MHCC97L and MHCC97H was sharply decreased (Figure 3D). These results combined with those of PU.1 knockdown indicated that PU.1 played a negative role in the invasion of hepatoma cells.

$P U .1$ was a transcription factor of tumor suppressor miR615-5p in hepatoma cell lines

We tried to explore the potential mechanism of PU.1 suppressing hepatoma cell invasion . An online platform ChIPBase, which decodes the regulatory relationship between transcription factors and non-coding RNAs/ proteins, showed that a newly identified tumor suppressor miR-615-5p was a target gene of PU.1 (Figure 4A). ChIP analysis was done to testify the target relationship between PU.1 and miR-615-5p promoter. The results showed that, compared to negative control (anti-IgG), PU.1 exhibited a high binding capacity (Figure 4B). Finally, we detected the effect of overexpression of PU.1 on the expression of miR-615-5p. Real-time qPCR analysis indicated that expression of pri-, pre- and mature miR-615-5p were all robustly upregulated by overexpression of PU.1 (Figure 4C). Western blotting analysis also showed the target gene of IGF2 was decreased by overexpression of PU.1 (Figure 4D).

These all above data revealed that PU.1 suppressed invasion of hepatoma cells through promoting miR-615$5 \mathrm{p}$.

\section{Discussion}

In this study, we found that levels of PU.1 mRNA in metastatic HCC tissues and metastatic HCC cell lines were significantly lower than those of adjacent noncancerous tissues and non- metastatic HCC cell lines. Then knockdown and overexpression analysis showed that PU.1 played a negative role in invasion of HCC cell lines. To our knowledge, it is the first report that PU.1 was identified to be a tumor suppressor in HCC. For a long time, PU.1 has been thought to be restricted in hematopoietic system (Loffreda et al., 1998; Pang et al., 2013; Wei et al., 2015). However, in last several years, evidence was gradually revealed that PU.1 functioned in various biological processes, not only in the hematopoiesis and immune system development but also in adipogenesis and insulin resistance (Lin et al., 2012). Our findings and the previous several reports indicated that PU.1 might play a broader range of roles in regulation of gene expression.

MiR-615-5p is a number of conserved miR-615 family which is located within the vertebrate Hox gene (Landgraf et al., 2007). MiR-615-5p was newly found and poorly characterized and we just knew very little about miR-615$5 p$ in several researches of small RNA library sequencing (Landgraf et al., 2007). In a most recent report, miR-615$5 p$ was proven to negatively regulate IGF- 2 in the posttranscriptional level and suppress proliferation, invasion and metastasis of HCC (El Tayebi et al., 2012). In this 
PU.1 Identified as a Novel Metastasis Suppressor in Hepatocellular Carcinoma Regulating miR-615-5p/IGF2 Axis

current study, ChIP-qPCR analysis and overexpression experiment showed that PU.1 was a transcription factor of miR-615-5p. According to a commonsense analysis, PU.1 should have an effect in suppression of proliferation, invasion and metastasis of HCC. But in fact, knockdown and overexpression of PU.1 both did not influenced the proliferation of HCC cell lines. We didn't know the exact reason. Whereas, owing to so many downstream genes controlled by PU.1, it was likely that other downstream genes regulated by PU.1 offset the inhibition of cell proliferation by miR-615-5p. The reason needs further studying.

In general, we identified PU.1 as an invasion suppressor in human HCC that is a non- hematopoietic system. PU.1 promoted expression of miR-615-5p suppressing invasion by negatively regulating IGF2. These findings improved our understanding of PU.1 regulatory roles and provided a potential target for metastatic HCC diagnosis and therapy.

\section{References}

Bruix J, Sherman M (2011). Management of hepatocellular carcinoma: an update. Hepatol, 53, 1020-2.

Christie DA, Xu LS, Turkistany SA, et al (2015). PU. 1 opposes IL-7-dependent proliferation of developing B cells with involvement of the direct target gene bruton tyrosine kinase. J Immunol, 194, 595-605.

Drusco A, Nuovo GJ, Zanesi N, et al (2014). MicroRNA profiles discriminate among colon cancer metastasis. PloS one, $\mathbf{9}$, 96670.

El Tayebi H, Hosny K, Esmat G, Breuhahn K, Abdelaziz AI (2012). MiR-615-5p is restrictedly expressed in cirrhotic and cancerous liver tissues and its overexpression alleviates the tumorigenic effects in hepatocellular carcinoma. FEBS Lett, 586, 3309-16.

Fornari F, Gramantieri L, Giovannini C, et al (2009). MiR-122/ cyclin G1 interaction modulates p53 activity and affects doxorubicin sensitivity of human hepatocarcinoma cells. Cancer Res, 69, 5761-7.

Guan J (2015). Cholesterol-conjugated let-7a miRNA mimics: promising tools for HCC systemic. RNA and DISEASE, 2, doi.org/10.14800/rd.630.

Kudo M, Matsui O, Izumi N, et al (2014). JSH consensusbased clinical practice guidelines for the management of hepatocellular carcinoma: 2014 update by the liver cancer study group of Japan. Liver Cancer, 3, 458-68.

Kutay H, Bai S, Datta J, et al (2006). Downregulation of miR122 in the rodent and human hepatocellular carcinomas. $J$ Cell Biochem, 99, 671-8.

Landgraf P, Rusu M, Sheridan R, et al (2007). A mammalian microRNA expression atlas based on small RNA library sequencing. Cell, 129, 1401-14.

Lin L, Pang W, Chen K, et al (2012). Adipocyte expression of PU. 1 transcription factor causes insulin resistance through upregulation of inflammatory cytokine gene expression and ROS production. Am J Physiol-Endoc M, 302, 1550-9.

Liu AM, Xu Z, Shek FH, et al (2014). miR-122 targets pyruvate kinase M2 and affects metabolism of hepatocellular carcinoma. PloS one, $9,86872$.

Llovet JM, Hernandez-Gea V (2014). Hepatocellular carcinoma: reasons for phase III failure and novel perspectives on trial design. Clin Cancer Res, 20, 2072-9.

Loffreda S, Yang S, Lin H, et al (1998). Leptin regulates proinflammatory immune responses. FASEB J, 12, 57-65.
Metcalf D, Dakic A, Mifsud S, et al (2006). Inactivation of PU. 1 in adult mice leads to the development of myeloid leukemia. Proc Natl Acad Sci USA, 103, 1486-91.

Olena AF, Patton JG (2014). MiRNA biogenesis and function. in: microRNA in development and in the progression of cancer, pp, 3-28.

Pang WJ, Lin LG, Xiong Y, et al (2013). Knockdown of PU. 1 AS lncRNA inhibits adipogenesis through enhancing PU. 1 mRNA translation. J Cell Biochem, 114, 2500-12.

Shimizu S, Takehara T, Hikita H, et al (2010). The let-7 family of microRNAs inhibits Bcl-xL expression and potentiates sorafenib-induced apoptosis in human hepatocellular carcinoma. J Hepatol, 52, 698-704.

Toiyama Y, Tanaka K, Kitajima T, et al (2014). Elevated serum angiopoietin-like protein 2 correlates with the metastatic properties of colorectal cancer: a serum biomarker for early diagnosis and recurrence. Clin Cancer Res, 20, 6175-86.

Wang F, Tong Q (2008). Transcription factor PU. 1 is expressed in white adipose and inhibits adipocyte differentiation. Am $J$ Physiol-Cell Ph, 295, 213-20.

Wei N, Pang W, Wang Y, et al (2014). Knockdown of PU. 1 mRNA and AS lncRNA regulates expression of immunerelated genes in zebrafish danio rerio. Dev Comp Immunol, 44, 315-9.

Wei N, Wang Y, Xu RX, et al (2015). PU. 1 antisense lncRNA against its mRNA translation promotes adipogenesis in porcine preadipocytes. Anim Genet, 46, 133-40. 